\title{
Diversidade florística e estoque de carbono de sistemas agroflorestais em dois municípios do nordeste paraense, Brasil
}

\author{
Floristic diversity and carbon stock of agroforestry systems in two
} municipalities of Northeast of State of Pará, Brazil

\author{
Silvio Roberto Miranda dos Santos', Osvaldo Ryohei Kato', Manoel Malheiros Tourinho' \\ 'Empresa Brasileira de Pesquisa Agropecuária. Belém, Pará, Brasil
}

\begin{abstract}
Resumo: Objetivou-se analisar a florística e o estoque de carbono (EC) de dois tipos de sistemas agroflorestais (SAF): tradicional, em Cametá, Pará (SAF-CM), e comercial, em Tomé-Açu, Pará (SAF-TA). Utilizaram-se parcelas de 50 m x 50 m, onde foram medidos o diâmetro à altura do peito (DAP) e a altura de todos os indivíduos $\left(n_{i}\right) \operatorname{com} D A P \geq 5 \mathrm{~cm}$. A biomassa seca dos $\mathrm{SAF}$ foi determinada pelo método indireto. Foram inventariados, em média, nos SAF-CM, $2.458 \mathrm{n}$. ha-1 ${ }^{-1}$, pertencentes a 12 famílias, 19 gêneros e 19 espécies, enquanto que, nos SAF-TA, 1.249 n. ha-1, pertencentes a nove famílias, 13 gêneros e 13 espécies. Euterpe oleracea (açaí) e Theobroma cacao (cacau) foram as espécies mais importantes nos SAF estudados. $O$ EC foi maior nas árvores do que no açaí e no cacau. O manejo adotado influenciou na riqueza de espécies e no EC. Os SAF-CM mostraram maior resiliência e os SAF-TA, maior antropia. Outras espécies destacaram-se em abundância e EC: Virola surinamensis, Hevea brasiliensis e Carapa guianensis, nos SAF-CM, e Spondias lutea, Bertholletia excelsa e Swietenia macrophylla, nos SAF-TA. Os resultados obtidos põem os SAF como alternativa de produção para consumo, venda e EC, ratificando a viabilidade socioeconômica e ambiental desses agroecossistemas na Amazônia.
\end{abstract}

Palavras-chave: Biodiversidade. Biomassa. Agroecossistemas. Amazônia.

Abstract: The objective of this study was to analyze the floristic and carbon stock (CS) of two types of agroforestry systems (SAF): traditional, in Cametá, Pará (SAF-CM), and commercial in Tomé-Açu, Pará (SAF-TA). We used plots of $50 \mathrm{~m} \times 50 \mathrm{~m}$, where the diameter at breast height $(\mathrm{DBH})$ and the height of all individuals $\left(\mathrm{n}_{\mathrm{i}}\right)$ with $\mathrm{DBH} \geq 5 \mathrm{~cm}$ were measured. The biomass of SAF was determined by the indirect method. They were inventoried on average in SAF-CM, 2,458 $\mathrm{n}_{i} \mathrm{ha}^{-1}$, belonging to 12 families, 19 genera and 19 species, while in SAF-TA, 1,249 $\mathrm{n}_{\mathrm{i}}$ ha ${ }^{-1}$, belonging to nine families, 13 genera and 13 species. Euterpe oleracea (açai) and Theobroma cacao (cacau) were the most important species in the SAF studied. CS was higher in trees than in açaí and cacau. The management adopted influenced the species richness and CS. The SAFCM showed higher resilience and the SAF-TA greater anthropy. Other species were found in abundance and CS: Virola surinamensis, Hevea brasiliensis and Carapa guianensis, in SAF-CM, and Spondias lutea, Bertholletia excelsa and Swietenia macrophylla, in SAF-TA. The results obtained put SAF as an alternative of production for consumption, sale and CS, ratifying the socioeconomic and environmental viability of these agroecosystems in the Amazon.

Keywords: Biodiversity. Biomass. Agroecosystems. Amazon.

SANTOS, S. R. M., O. R. KATO \& M. M. TOURINHO, 2019. Diversidade florística e estoque de carbono de sistemas agroflorestais em dois municípios do nordeste paraense, Brasil. Boletim do Museu Paraense Emílio Goeldi. Ciências Naturais 14(1): 31-42.

Autor para correspondência: Silvio Roberto Miranda dos Santos. Universidade Federal Rural da Amazônia. Instituo Socioambiental e dos Recursos Hídricos (ISARH). Projeto Várzea. Avenida Presidente Tancredo Neves, 2501 - Terra Firme. Belém, PA, Brasil. CEP 66077-830 (silvio.santos@ufra.edu.br, silviormsantos@gmail.com).

Recebido em 11/05/2017

Aprovado em 16/11/2018

Responsabilidade editorial: Fernando da Silva Carvalho Filho
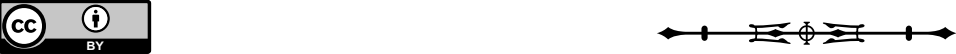


\section{INTRODUÇÃO}

O desenvolvimento econômico mundial sempre esteve baseado na premissa da infinita oferta de recursos naturais e na suposição da capacidade do homem de manejar a natureza sem comprometê-la (Santos et al., 2004). Porém, diante dessas suposições, vem acontecendo o desequilíbrio ambiental, atingindo a vida, em amplo sentido, assim como as atividades econômicas, tornando-se imperiosa a análise dos ônus ambientais, ecológicos e sociais decorrentes do uso inadequado desses recursos (Sougata \& Shibu, 2012).

Nesse contexto de ofertas de alternativas de uso e de recuperação dos solos, por exemplo, colocam-se os sistemas agroflorestais (SAF), que são uma forma de uso da terra que combina o cultivo de espécies arbóreas, arbustivas, agrícolas e pastagens, em função do tempo e do espaço, visando à produção e ao desenvolvimento de forma sustentável (Fassbender, 1993). Eles favorecem a resiliência e a estabilidade ambiental (Porro et al., 2012), como também estimulam o incremento em biomassa (Osterrooht, 2002), colocando-se, assim, como um dos sistemas de uso da terra capaz de contribuir para a produção, a renda e a estocagem de carbono, reduzindo, via fotossíntese, os danos provocados pelo gás carbônico $\left(\mathrm{CO}_{2}\right)$, um dos principais causadores de efeito estufa (Dias et al., 2015; Villa et al., 2015).

Segundo Homma et al. (1995), os SAF geram estabilidade econômica, produzindo bens para consumo e para venda, razão pela qual, com seu uso, haveria uma tendência de reduzir a fronteira agrícola e o avanço em áreas de florestas, contribuindo para a melhoria da qualidade ambiental. Eles também se constituem em uma das alternativas de sistemas assimiladores e acumuladoes de carbono na biomassa vegetal e no solo (Kato el al., 2006).

A quantificação de biomassa de um ecossistema é comumente referida como essencial aos estudos de carbono. A riqueza florística impacta diretamente nessa biomassa, podendo ser determinada pelos métodos direto (destrutivo) e indireto (não destrutivo). O primeiro é mais acurado, sendo, contudo, difíil cortar e pesar todas árvores em uma área, enquanto o segundo é mais rápido, pois utiliza variáveis facilmente obtidas nas pesquisas de campo, como o diâmetro e a altura (Higuchi et al., 1998; Hairiah et al., 2001). Todavia, a biomassa deve ser gerada de forma fidedigna para que haja consistência nas suas quantificação e avaliação (Hairiah et al., 2001; Dallagnol et al., 2011).

Estudos mostram que é crescente o número de plantios em SAF na Amazônia, os quais podem apresentar várias classificações e tipos, podendo variar mais, devido à dinâmica de sucessão e à diversidade vegetal. Smith et al. (1998) classificaram os SAF em tradicional e comercial. $\bigcirc$ SAF-tradicional caracteriza-se por apresentar alta diversidade de espécies, maior uso de regeneração natural, mais espécies para consumo, menor uso de insumos e de mão de obra, sendo aquele praticado por comunidades indígenas e tradicionais; já o SAF-comercial caracteriza-se pela baixa diversidade de espécies, menor uso da regeneração natural, mais espécies para venda, maior uso de insumos e de mão de obra, sendo praticado sob orientação e assistência técnica.

Considerando-se que os SAF desempenham importante papel no manejo dos recursos naturais, contribuindo para a redução de agravos ambientais causados por desmatamentos e queimadas, sendo, ainda, meio eficaz para estocar carbono, este artigo teve como objetivos analisar os padrões de riqueza florística e determinar o estoque de carbono (EC) de SAF com diferentes práticas de manejo nos municípios de Cametá e de Tomé-Açu, no estado do Pará, Brasil.

\section{MATERIAL E MÉTODOS}

Este estudo foi realizado nos municípios de Cametá e Tomé-Açu, ambos pertencentes à mesorregião do nordeste paraense (Figura 1). $\bigcirc$ clima, conforme a classificação de Köppen, nos dois municípios, é do tipo Ami, tropical, quente e úmido. Cametá situa-se a $184 \mathrm{~km}$ a sudoeste de Belém (02 14' 40" Se 49०29' 45” W), com área de 3.122,9km². A temperatura média anual é de $26,5^{\circ} \mathrm{C}$, com mínima de $22^{\circ} \mathrm{C}$ e máxima de $31^{\circ} \mathrm{C}$; a umidade relativa do ar é de $85 \%$; a pluviosidade anual é elevada, entre $2.250 \mathrm{~mm}$ a 2.500 mm, com média de 2.375 mm, maior entre janeiro 
a maio e menor entre junho a dezembro. Tomé-Açu, por sua vez, situa-se a 208 km ao sul de Belém (02 40' 54" S e 480 16' 11" W), com área de 5.145,4 km². A temperatura média anual é de $26,8^{\circ} \mathrm{C}$, com mínima de $21^{\circ} \mathrm{C}$ e máxima de $33^{\circ} \mathrm{C}$; a umidade relativa do ar é de $85 \%$; a pluviosidade anual é elevada, entre $2.144 \mathrm{~mm}$ a $2.581 \mathrm{~mm}$, com média de $2.362 \mathrm{~mm}$, maior entre dezembro a maio e menor entre junho a novembro (IBGE, 2012).

O SAF-tradicional é o que melhor caracteriza o que foi verificado em Cametá, uma vez que esses sistemas são originados de capoeiras (enriquecidas com espécies frutíferas), sendo submetidos a manejo moderado e esporádico, que visa minimizar impactos no ambiente e na área de produção. Ele consiste no desbaste de açaizeiros (corte das palmeiras mais velhas cuja produção de frutos está reduzindo, as quais, no entanto, são aproveitadas na produção de palmito), cortes de cipós e de espécies indesejáveis e invasoras, coletas de sementes, frutos, óleos, casca e ervas medicinais e eventual extração de madeira para uso na propriedade. Esses SAF têm aparência de uma agrofloresta, em razão de apresentarem povoamento vegetal permanente, cuja criação está quase sempre ligada às atividades agrícolas e/ou extrativistas, evidenciando composição diversificada que reúne em uma mesma área

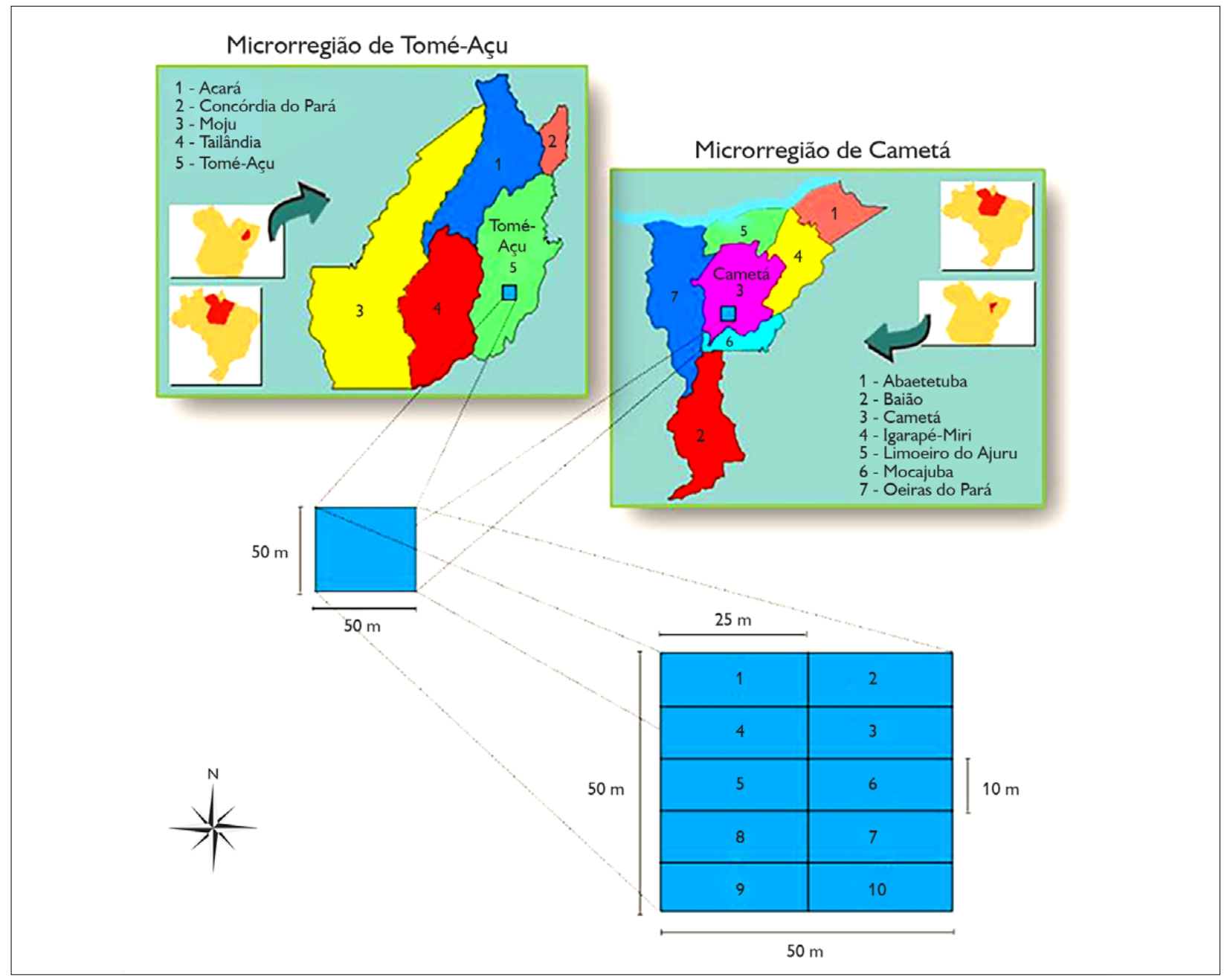

Figura 1. Áreas dos estudos nos municípios de Cametá e de Tomé-Açu, estado do Pará, Brasil. Mapa: S. R. M. Santos (2018).

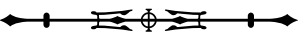


espécies arbóreas, arbustivas e frutíferas, formando pelo menos três níveis de copas e sub-bosque (Dubois, 1996).

O SAF-comercial, por sua vez, é o que melhor caracteriza o estudado em Tomé-Açu. Esses sistemas são os mais cultivados entre os agricultores nipo-brasileiros da região, sendo vistos como referência de eficiência técnica, produtidade e viabilidade econômica, submetidos a manejo intenso, a fim de se obter raleamento da área para incremento de espécies como cacau e açaí, importantes para a venda. Começaram a ser implantados em substituição aos pimentais, praticamente dizimados pela fusariose, doença causada pelo fungo Fusarium solani f. sp. piperis, após o colapso na produção ocorrido entre os anos de 1950 e 1970 (Homma, 2006). Apresentam algumas particularidades na sua origem e/ou implantação, como a sequência de plantio: pimenta-do-reino (Piper nigrum L.) $>>>$ maracujá (Passiflora edulis Sims) $>>>$ frutíferas arbustivas $>>>$ frutíferas palmeiras $>>$ frutíferas arbóreas $>>>$ espécies florestais.

Foram selecionados oito SAF, quatro em Cametá (SAF-CM) - SAF.1-CM, SAF.2-CM, SAF.3-CM e SAF.4-CM - e quatro em Tomé-Açu (SAF-TA) - SAF.1-TA, SAF.2-TA, SAF.3-TA e SAF.4-TA (Figura 1). A média de idade desses SAF nas duas áreas de estudo foi de 12 anos. Nessas unidades, foram inventariadas três parcelas amostrais de $50 \mathrm{mx}$ $50 \mathrm{~m}$ (0,25 ha), em um total de doze parcelas, divididas em dez subparcelas de $10 \mathrm{~m} \times 25 \mathrm{~m}$ (para orientar o sentido de medição), onde foram medidos, com fita diamétrica a 1,30 m do solo e com vara de altura, respectivamente, o diâmetro à altura do peito (DAP) e a altura total $(H)$ de todos os indivíduos com DAP $\geq 5 \mathrm{~cm}$. Todos os dados foram anotados em planilha de campo.

A análise florística foi realizada por meio da identificação de espécies, nos SAF, pelos nomes comuns conferidos por parataxonomistas. Em relação às não identificadas, coletou-se material botânico para que fossem identificadas no herbário da Universidade Federal Rural da Amazônia. A análise estrutural seguiu as recomendações de Brower et al. (1998), avaliada pela abundância ( $N=n_{i}$, onde: $n_{i}$ é o número de indivíduos da espécie), calculada em indivíduo ( $\left.n_{i}\right)$ por unidade de área (hectare $=$ ha). Então, temos: indivíduo por hectare $\left(n_{i}, a^{-1}\right)$.

Para determinar a biomassa seca (BS) acima do solo, a vegetação dos SAF foi dividida em três componentes principais: açaí, cacau e árvores. A BS foi calculada pelo método indireto, utilizando as equações alométricas de Bartelt et al. (2000): as equações 1, 3 e 5 determinam BS de folhas, 2 determina BS de estipes e 4 e 6 determinam BS do lenho, sendo que o termo lenho engloba as partes aéreas da planta - fuste, galhos e cascas (Tabela 1). A BS foi calculada em unidade de peso (megagrama $=\mathrm{Mg}$ ) por unidade de área (hectare $=$ ha), para depois, assim, determinar o carbono (C) contido na BS ( $\mathrm{Mg} C$ ha-1). O EC contido na BS, em áreas naturais ou antrópicas, tem sido obtido pela multiplicação da BS total (BS contida em cada parte da planta) por um fator igual a 0,45 , pois, em média, a BS contém, aproximadamente, $45 \%$ de carbono, entre 0,43 a 0,48 (Murillo, 1997; Higuchi et al., 1998; Thibau, 2000; Montagnini \& Nair, 2004). Neste estudo, optou-se pelo fator 0,45 , por ter sido observado em pesquisas com espécies nativas da Amazônia (Higuchi \& Carvalho Jr., 1994; Higuchi et al., 1998).

Como é imperativo comparar os resultados de um estudo com os de outros já realizados, no caso do presente artigo, os resumos de resultados estão contidos na Tabela 2. Assim, visando minorar distorções na aferição dos estudos, elaborou-se três categorias de uso da terra, onde os ecossistemas foram agrupados conforme os níveis de antropia e de manejo: a) ecossistemas antrópicos: aqueles que foram plantados e têm manejo constante e intensivo, como os SAF-TA; b) ecossistemas antropizados: aqueles que já foram explorados e/ou alterados, têm manejo moderado e esporádico, com vistas à recuperação ambiental e à viabilidade comercial, como os SAF-CM; e c) ecossistemas naturais: aqueles que não foram explorados e/ou alterados, têm pequenas intervenções e uso eventual, apresentam extrativismo de produtos não madeireiros, mas, mesmo assim, mantêm sua fisionomia e características naturais. 
Tabela 1. Equações utilizadas para determinar a biomassa seca (BS) acima do solo na vegetação em sistemas agroflorestais (SAF), estudados em Cametá e em Tomé-Açu, Pará. Legendas: coeficiente de determinação = R²; diâmetro à altura do peito = DAP. São apresentadas, respectivamente, as equações 1 e 2 (BS-folhas e BS-estipes de açaí), 3 e 4 (BS-folhas e BS-lenho de cacau) e 5 e 6 (BS-folhas e BS-lenho de árvores). Nas equações 4 e 6, o termo lenho engloba as partes aéreas da planta: fuste, galhos, casca. Fonte: Bartelt et al. (2000).

\begin{tabular}{|c|c|c|c|}
\hline & Equações alométricas & $\mathrm{R}^{2}$ & Nível da amostra (cm) \\
\hline \multicolumn{4}{|c|}{ Açaí } \\
\hline 1 & $\mathrm{BS}=\exp (-0,0550+0,0451 \times \mathrm{DAP})$ & 0,92 & $\mathrm{DAP} \geq 5$ \\
\hline 2 & $\mathrm{BS}=\exp (-0,0470+0,0750 \times \mathrm{DAP})$ & 0,99 & $\mathrm{DAP} \geq 5$ \\
\hline \multicolumn{4}{|c|}{ Cacau } \\
\hline 3 & $\mathrm{BS}=\exp (-1,3200+0,0566 \times \mathrm{DAP})$ & 0,96 & $\mathrm{DAP} \geq 5$ \\
\hline 4 & $\mathrm{BS}=\exp (0,0320+0,0810 \times \mathrm{DAP})$ & 0,99 & $\mathrm{DAP} \geq 5$ \\
\hline \multicolumn{4}{|c|}{ Árvores } \\
\hline 5 & $\mathrm{BS}=\exp (-0,7600+0,1242 \times \mathrm{DAP})$ & 0,77 & $\mathrm{DAP} \geq 5$ \\
\hline 6 & $\mathrm{BS}=\exp (0,2040+0,3129 \times \mathrm{DAP})$ & 0,99 & $\mathrm{DAP} \geq 5$ \\
\hline
\end{tabular}

Tabela 2. Resumo de médias de estoque de carbono (EC) na biomassa seca (BS) acima do solo encontrado em diversos sistemas de uso da terra na Amazônia: sistema agrofloretal (SAF), quintal agroflorestal (QAF), floresta de várzea (FVA), floresta de terra firme (FTF), capoeiras $(C A P)$, capoeiras enriquecidas (CAE), consórcios de árvores (CAR), monocultivos de árvores (MCA) e pastagens (PAS). Legenda: * = valores médios calculados a partir dos dados originais dos referidos estudos utilizando o fator de conversão de carbono $(0,45)$, adaptados pelos autores deste estudo.

\begin{tabular}{|c|c|c|c|c|c|}
\hline- & $\begin{array}{c}\mathrm{BS} \\
\left(\mathrm{Mg} \mathrm{ha}^{-1}\right)\end{array}$ & $\begin{array}{c}\mathrm{EC} \\
(\mathrm{Mg} \mathrm{Cha-1})^{*}\end{array}$ & Tipos & Local & Fonte \\
\hline \multirow{6}{*}{ 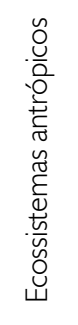 } & 184,40 & 82,98 & QAF & Arari - Maranhão & Mavisoy (2013) \\
\hline & 57,80 & 26,01 & PAS & Anajatuba - Maranhão & Mavisoy (2013) \\
\hline & 89,10 & 40,10 & SAF & Tomé-Açu - Pará & Bolfe et al. (2012) \\
\hline & 78,00 & 35,10 & CAR & Igarapé-Açu - Pará & Brienza Júnior (2012) \\
\hline & 166,67 & 75,00 & MCA & Manaus - Amazonas & Chaves et al. (2011) \\
\hline & 70,83 & 31,87 & CAR & Porto Velho - Rondônia & Locatelli et al. (2004) \\
\hline \multirow{6}{*}{ 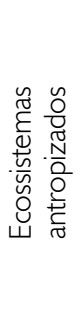 } & 84,80 & 38,16 & CAE & Morros - Maranhão & Mavisoy (2013) \\
\hline & 196,64 & 88,49 & CAP & Nova Ipixuna - Pará & Costa et al. (2012) \\
\hline & 181,31 & 81,59 & CAP & Pacajá - Pará & Costa et al. (2012) \\
\hline & 68,16 & 30,67 & CAP & Parauapebas - Pará & Costa et al. (2012) \\
\hline & 199,00 & 89,55 & CAE & Paragominas - Pará & Pereira (2001) \\
\hline & 106,00 & 47,70 & CAE & Igarapé-Açú - Pará & Brienza Júnior et al. (2001) \\
\hline \multirow{6}{*}{ 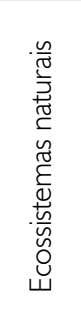 } & 255,91 & 115,16 & FTF & Nova Ipixuna - Pará & Costa et al. (2012) \\
\hline & 346,06 & 155,73 & FTF & Manaus - Amazonas & Matos \& Kirchner (2008) \\
\hline & 246,10 & 110,74 & FVA & Cametá - Pará & Bartelt et al. (2000) \\
\hline & 307,00 & 138,15 & FVA & Abaetetuba - Pará & Tsuchiya \& Hiraoka (1999) \\
\hline & 289,00 & 130,05 & FTF & Abaetetuba - Pará & Tsuchiya \& Hiraoka (1999) \\
\hline & 428,00 & 192,60 & FTF & Floresta amazônica & Fearnside (1996) \\
\hline
\end{tabular}




\section{RESULTADOS E DISCUSSÃO}

Nos SAF estudados em Cametá (SAF-CM), foram inventariados, em média, $2.458 \mathrm{n}_{\mathrm{i}} \mathrm{ha}^{-1}$, com DAP $\geq 5 \mathrm{~cm}$, pertencentes a 12 famílias, 19 gêneros e 19 espécies; Mimosaceae foi a família com maior número de espécies $(i=3)$. Nos SAF estudados em Tomé-Açu (SAF-TA), foram inventariados, em média, $1.249 \mathrm{n}_{\mathrm{i}} \mathrm{ha}^{-1}$, com DAP $\geq 5 \mathrm{~cm}$, pertencentes a nove famílias, 13 gêneros e 13 espécies; Anacardiaceae foi a família com maior número de espécies ( $i=3$ ) (Tabela 3). Nos SAF-CM, cerca de $68,4 \%$ das espécies são para consumo e $52,6 \%$ para venda; enquanto nos SAF-TA, 46,2\% são para consumo e 92,3\% para venda (Tabela 3). Os percentuais excedem 100\%, haja vista a existência de espécies multiuso. Nos SAF estudados em Igarapé-Açu, no Pará, por Vieira et al. (2007), cerca de $25 \%$ das espécies foram declaradas para consumo e 93\% para venda; essas taxas são inferiores às encontradas nos SAF-CM e SAF-TA para consumo e superiores às encontradas nos SAF-CM, sendo, ainda, compatíveis às encontradas nos SAF-TA para venda.

A riqueza encontrada nos SAF-CM $(i=19)$ foi superior à encontrada nos SAF-TA $(i=13)$, diferença relativa ao percentual $(89,5 \%)$ de espécies nativas de regeneração espontânea mantidas nos SAF-CM, onde seis espécies (31,6\%) foram comuns a todos os SAF: Euterpe oleracea, Theobroma cacao, Virola surinamensis, Hevea brasiliensis, Carapa guianensis e Inga paraensis; enquanto nos SAF-TA, apenas duas $(15,4 \%)$ foram comuns: $E$. oleracea e T. cacao. Nesses SAF, outras espécies se destacaram: Bertholletia excelsa, Swietenia macrophylla e Spondias lutea (Tabela 3). Observou-se, com o passar do tempo e com a maturação dos SAF, redução no número de espécies: $26,7 \%$ nos SAF-CM e 50\% nos SAF-TA. Porém, a riqueza de espécies em SAF está relacionada ou condicionada ao arranjo, ao objetivo e ao manejo adotados: moderado, priorizando as espécies para consumo na propriedade (segurança alimentar), como nos SAF-CM, ou intenso, visando favorecer as espécies de maior valor comercial, como nos SAF-TA.
A riqueza encontrada neste estudo foi superior à verificada em outras pesquisas, como nos SAF $(i=9)$ na Amazônia oriental (Mavisoy, 2013), nos SAF ( $i=10)$ em Tomé-Açu (Brancher, 2010) e nos quintais agroflorestais $(i=10)$ nas várzeas de Bragança (Bentes-Gama et al., 1999); inferior em relação à encontrada nos SAF ( $i=$ 29 e 54) em Tomé-Açu (Bolfe et al., 2009, 2012) e nos SAF ( $\mathrm{i}=23$ ) das várzeas de Afuá (Queiroz \& Mochiutti, 2000); e equidiferente e compatível com a encontrada nos SAF ( $i=16$ ) em Igarapé-Açu (Vieira et al., 2007). As espécies mais importantes neste estudo, E. oleraceae e T. cacao, também foram as mais destacadas em outros realizados na Amazônia (Mavisoy, 2013; Bolfe et al., 2012; Kato et al., 2012; Brancher, 2010; Oliveira \& Kato, 2010).

Para determinar o EC dos SAF, a vegetação foi dividida em açaí, cacau e árvores. Assim, a abundância nos SAF-CM foi estabelecida em açaí (53,0\%), cacau (29,8\%) e árvores (17,2\%) e, nos SAF-TA, em açaí (26,6\%), cacau (65,8\%) e árvores $(7,6 \%)$ (Tabela 3).

A média de EC nos SAF-CM foi de 57,45 Mg C ha-1, sendo: $1,95 \mathrm{Mg} \mathrm{C} \mathrm{ha}^{-1}$ no açaí (42,8\% nas folhas e 57,2\% nos estipes), 0,72 Mg C ha ${ }^{-1}$ no cacau (17,9\% nas folhas e $82,1 \%$ no lenho) e $54,77 \mathrm{Mg} C$ ha $^{-1}$ nas árvores (1\% nas folhas e 99\% no lenho); enquanto, nos SAF-TA, foi de 24,07 Mg C ha-1, sendo: 0,53 Mg C ha-1 no açaí (42,2\% nas folhas e 57,8\% nos estipes), 1,15 Mg C ha-1 no cacau (16,2\% nas folhas e 83,8\% no lenho) e 22,39 Mg C ha-1 nas árvores (0,7\% nas folhas e 99,3\% no lenho) (Tabela 4).

Entre as árvores, algumas se destacaram em EC, como C. guianensis, V. surinamensis e H. brasiliensis, presentes nos quatro SAF-CM. Assim, V. surinamensis e $H$. brasiliensis apresentaram EC de, respectivamente, 23,53 Mg C ha-1 e 19,19 Mg C ha-1 no SAF.1-CM e 23,30 $\mathrm{MgC} \mathrm{ha-1}$ e 22,93 Mg C ha-1 no SAF.4-CM, Juntas, essas espécies representaram $52,4 \%$ do EC total do SAF.1CM e $72,8 \%$ do EC total do SAF.4-CM. Além disso, $C$. guianensis manteve o EC estável nos quatro SAF-CM, com média de 10,76 Mg C ha-1 (Figura 2). 
Tabela 3. Espécies encontradas em sistemas agroflorestais (SAF), estudados em Cametá (SAF-CM) e em Tomé-Açu (SAF-TA), Pará. Legendas: $\mathrm{N}=$ abundância; finalidade das plantas $-\mathrm{C}=$ consumo $\mathrm{e}=\mathrm{V}=$ venda.

(Continua)

\begin{tabular}{|c|c|c|c|c|c|c|c|}
\hline \multirow{3}{*}{$\begin{array}{l}\text { Família/Espécie } \\
\text { Anacardiaceae }\end{array}$} & \multirow{3}{*}{ Nome vulgar } & \multicolumn{4}{|c|}{$N\left(n_{i} h a^{-1}\right)$} & \multirow{2}{*}{\multicolumn{2}{|c|}{ Finalidade (\%) }} \\
\hline & & \multirow[t]{2}{*}{ SAF.1-CM } & \multirow[t]{2}{*}{ SAF.2-CM } & \multirow[t]{2}{*}{ SAF.3-CM } & \multirow[t]{2}{*}{ SAF.4-CM } & & \\
\hline & & & & & & & \\
\hline Anacardium giganteum W. Hancock ex Engl. & Cajuí & - & 20 & 16 & - & & $\vee$ \\
\hline Spondias lutea L. & Taperebá & 16 & - & - & 12 & C & $\vee$ \\
\hline \multicolumn{8}{|l|}{ Arecaceae } \\
\hline Euterpe oleracea Mart. & Açaí & 1.872 & 1.484 & 1.352 & 504 & C & $\mathrm{V}$ \\
\hline Mauritia flexuosa L. f. & Buriti & 28 & - & 16 & - & C & \\
\hline \multicolumn{8}{|l|}{ Caesalpiniaceae } \\
\hline Macrolobium angustifolium (Benth.) R.S. Cowan & Ipê-da-várzea & 12 & 8 & - & - & & $\vee$ \\
\hline Swartzia acuminata Willd. ex Vogel & Pitaíca & 16 & 8 & - & - & C & \\
\hline \multicolumn{8}{|l|}{ Clusiaceae } \\
\hline Symphonia globulifera L. f. & Anani & 16 & - & - & - & & $\mathrm{V}$ \\
\hline \multicolumn{8}{|l|}{ Euphorbiaceae } \\
\hline Hura crepitans L. & Assacu & - & 16 & - & 12 & & $\vee$ \\
\hline Hevea brasiliensis (Willd. ex A. Juss.) Müll. Arg. & Seringueira & 104 & 64 & 36 & 120 & & $\vee$ \\
\hline \multicolumn{8}{|l|}{ Fabaceae } \\
\hline Pterocarpus amazonicus Huber & Mututi & 44 & 16 & - & 16 & C & \\
\hline \multicolumn{8}{|l|}{ Meliaceae } \\
\hline Carapa guianensis Aubl. & Andiroba & 68 & 108 & 72 & 60 & C & $\vee$ \\
\hline \multicolumn{8}{|l|}{ Mimosaceae } \\
\hline Pithecellobium acacioides Ducke & Esponjeira & - & - & 8 & 16 & C & \\
\hline Inga paraensis Ducke & Ingá & 12 & 8 & 16 & 8 & C & \\
\hline Pentaclethra macroloba (Willd.) Kuntze & Pracaxi & 12 & 16 & 12 & - & C & \\
\hline \multicolumn{8}{|l|}{ Moraceae } \\
\hline Mora paraensis Ducke & Pracuúba & - & 12 & 12 & 12 & C & \\
\hline \multicolumn{8}{|l|}{ Myristicaceae } \\
\hline Virola surinamensis (Rol. ex Rottb.) Warb. & Virola & 228 & 124 & 84 & 172 & & $\vee$ \\
\hline \multicolumn{8}{|l|}{ Sapotaceae } \\
\hline Sarcaulus brasiliensis (A. DC.) Eyma & Jaraí-branco & 8 & 8 & - & - & C & \\
\hline \multicolumn{8}{|l|}{ Sterculiaceae } \\
\hline Theobroma cacao L. & Cacau & 68 & 496 & 984 & 1.380 & C & $\mathrm{V}$ \\
\hline Sterculia speciosa K. Schum. & Capoteiro & 8 & - & 12 & - & C & \\
\hline \multicolumn{2}{|l|}{ Total $\left(\right.$ média $\left.=2.458 \mathrm{n}_{\mathrm{i}} \mathrm{ha}^{-1}\right)$} & 2.512 & 2.388 & 2.620 & 2.312 & 68,4 & 52,6 \\
\hline \multirow{2}{*}{ Família/Espécie } & \multirow{2}{*}{ Nome vulgar } & & $N\left(n_{i}\right.$ & $\left.h a^{-1}\right)$ & & \multirow{2}{*}{\multicolumn{2}{|c|}{ Finalidade (\%) }} \\
\hline & & SAF.1-TA & SAF.2-TA & SAF.3-TA & SAF.4-TA & & \\
\hline \multicolumn{8}{|l|}{ Anacardiaceae } \\
\hline Astronium lecointei Ducke & Aroeira & 16 & - & - & - & & $\vee$ \\
\hline Anacardium giganteum W. Hancock ex Engl. & Cajuí & 4 & - & - & - & & $\vee$ \\
\hline Spondias lutea L. & Taperebá & - & - & 16 & 63 & C & $\vee$ \\
\hline Arecaceae & & & & & & & \\
\hline
\end{tabular}


Tabela 3.

(Conclusão)

\begin{tabular}{|c|c|c|c|c|c|c|c|}
\hline \multirow{3}{*}{$\begin{array}{c}\text { Família/Espécie } \\
\text { Euterpe oleracea Mart. }\end{array}$} & \multirow{3}{*}{$\begin{array}{c}\text { Nome vulgar } \\
\text { Açaí }\end{array}$} & \multicolumn{4}{|c|}{$N\left(n_{i} h a^{-1}\right)$} & \multirow{2}{*}{\multicolumn{2}{|c|}{ Finalidade (\%) }} \\
\hline & & \multirow{2}{*}{$\begin{array}{c}\text { SAF.1-TA } \\
171\end{array}$} & \multirow{2}{*}{$\begin{array}{c}\text { SAF.2-TA } \\
95\end{array}$} & \multirow{2}{*}{$\begin{array}{c}\text { SAF.3-TA } \\
543\end{array}$} & \multirow{2}{*}{$\begin{array}{c}\text { SAF.4-TA } \\
520\end{array}$} & & \\
\hline & & & & & & C & $\mathrm{V}$ \\
\hline \multicolumn{8}{|l|}{ Bignoniaceae } \\
\hline Tabebuia serratifolia (Vahl) G. Nicholson & Ipê-amarelo & 13 & 5 & - & - & & $\mathrm{V}$ \\
\hline \multicolumn{8}{|l|}{ Caesalpiniaceae } \\
\hline Schizolobium amazonicum Huber ex Ducke & Paricá & 11 & 5 & - & - & & $\vee$ \\
\hline Clitoria fairchildiana R.A. Howard & Palheteira & - & - & - & 20 & & \\
\hline \multicolumn{8}{|l|}{ Lecythidaceae } \\
\hline Bertholletia excelsa Bonpl. & $\begin{array}{c}\text { Castanha-do- } \\
\text { pará }\end{array}$ & 3 & 40 & - & - & C & $\vee$ \\
\hline \multicolumn{8}{|l|}{ Meliaceae } \\
\hline Carapa guianensis Aubl. & Andiroba & - & 32 & - & - & & $\mathrm{V}$ \\
\hline Swietenia macrophylla King & Mogno & 55 & - & 40 & - & & $\vee$ \\
\hline \multicolumn{8}{|l|}{ Musacea } \\
\hline Musa sapientum $\mathrm{L}$. & Bananeira & - & - & 49 & - & C & $\mathrm{V}$ \\
\hline \multicolumn{8}{|l|}{ Sapindaceae } \\
\hline Nephelium lappaceum L. & Rambutã & - & 7 & - & - & C & $\vee$ \\
\hline \multicolumn{8}{|l|}{ Sterculiaceae } \\
\hline Theobroma cacao L. & Cacau & 1.044 & 472 & 1.123 & 651 & C & $\mathrm{V}$ \\
\hline \multicolumn{2}{|l|}{ Total $\left(\right.$ média $\left.=1.249 \mathrm{n}_{\mathrm{i}} \mathrm{ha}^{-1}\right)$} & 1.316 & 656 & 1.771 & 1.253 & 46,2 & 92,3 \\
\hline
\end{tabular}

Tabela 4. Estoque de carbono (EC) acima do solo, encontrado em sistemas agroflorestais (SAF), estudados em Cametá (SAF-CM) e em Tomé-Açu (SAF-TA), Pará. Média anual de EC (MA), médias (M) e erro padrão da média (e). Nas equações (Eq) 4 e 6, o termo lenho engloba as partes aéreas da planta: fuste, galhos, casca.

\begin{tabular}{|c|c|c|c|c|c|c|c|c|c|c|c|c|}
\hline \multirow{3}{*}{$\begin{array}{l}\text { Equações } \\
\text { alométricas }\end{array}$} & \multicolumn{12}{|c|}{ EC $\left(M g C h a^{-1}\right)$} \\
\hline & \multicolumn{6}{|c|}{ SAF-CM } & \multicolumn{6}{|c|}{ SAF-TA } \\
\hline & 1 & 2 & 3 & 4 & $M$ & e & 1 & 2 & 3 & 4 & $M$ & e \\
\hline \multicolumn{13}{|c|}{ Açaí } \\
\hline Eq-1 (folhas) & 1,20 & 0,96 & 0,86 & 0,33 & 0,84 & 0,02 & 0,11 & 0,06 & 0,36 & 0,36 & 0,22 & 0,02 \\
\hline Eq-2 (estipes) & 1,61 & 1,28 & 1,15 & 0,45 & 1,12 & 0,03 & 0,14 & 0,08 & 0,50 & 0,50 & 0,30 & 0,03 \\
\hline Total & 2,81 & 2,24 & 2,02 & 0,78 & 1,96 & 0,05 & 0,24 & 0,14 & 0,86 & 0,86 & 0,53 & 0,05 \\
\hline \multicolumn{13}{|c|}{ Cacau } \\
\hline Eq-3 (folhas) & 0,01 & 0,09 & 0,18 & 0,24 & 0,13 & 0,01 & 0,20 & 0,13 & 0,27 & 0,16 & 0,19 & 0,00 \\
\hline Eq-4 (lenho) & 0,05 & 0,40 & 0,83 & 1,10 & 0,59 & 0,04 & 0,91 & 0,73 & 1,39 & 0,83 & 0,97 & 0,02 \\
\hline Total & 0,07 & 0,48 & 1,00 & 1,34 & 0,72 & 0,05 & 1,11 & 0,85 & 1,66 & 0,99 & 1,15 & 0,03 \\
\hline \multicolumn{13}{|c|}{ Árvores } \\
\hline Eq-5 (folhas) & 0,78 & 0,45 & 0,42 & 0,60 & 0,56 & 0,02 & 0,12 & 0,17 & 0,16 & 0,22 & 0,17 & 0,01 \\
\hline Eq-6 (lenho) & 77,88 & 35,71 & 42,41 & 60,81 & 54,20 & 2,05 & 10,24 & 22,75 & 24,92 & 30,99 & 22,22 & 1,99 \\
\hline Total & 78,65 & 36,17 & 42,83 & 61,41 & 54,77 & 2,07 & 10,36 & 22,91 & 25,08 & 31,21 & 22,39 & 2,00 \\
\hline Total & 81,53 & 38,89 & 45,85 & 63,53 & 57,45 & 2,17 & 11,71 & 23,91 & 27,60 & 33,06 & 24,07 & 2,08 \\
\hline$M A\left(\mathrm{Mg} \mathrm{C} \mathrm{Ha}^{-1} \mathrm{Ano}{ }^{1}\right)$ & 8,15 & 3,54 & 3,53 & 4,54 & 4,94 & 0,04 & 3,90 & 2,66 & 1,84 & 1,57 & 2,49 & 0,03 \\
\hline
\end{tabular}


Nos SAF-TA, contudo, destacaram-se em EC: $S$. lutea, B. excelsa e Clitoria fairchildiana. Especialmente S. lutea com EC de 48,96 $\mathrm{Mg} \mathrm{C} \mathrm{ha-1}(66,6 \%)$ no SAF.4-TA e 17,55 Mg C ha-1 (63,6\%) no SAF.3-TA, que sozinha representou $41,1 \%$ do EC total dos quatro SAFTA. Outras espécies também mereceram destaque: Schizolobium amazonicum, com EC de 6,22 Mg C ha-1
(53,1\%), e S. macrophylla, com EC de 3,56 Mg C ha-1 $(14,9 \%)$ no SAF.1-TA (Figura 3). Os resultados acima de EC credenciam essas espécies para compor os SAF e/ou outros sistemas produtivos nos referidos municípios, em regiões adjacentes e também em relação à Amazônia, independente de qual seja sua finalidade (se comercial, consumo doméstico ou ambiental).

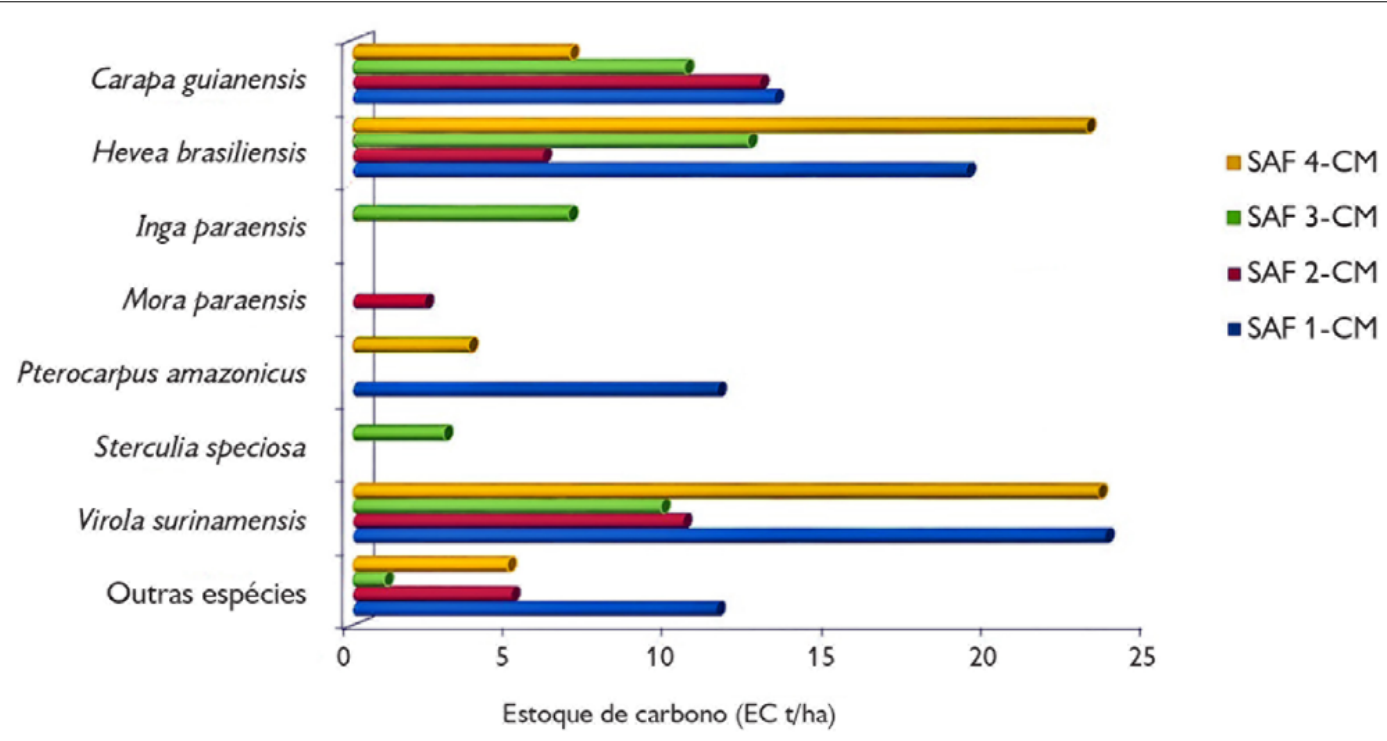

Figura 2. Estoque de carbono (EC) na biomassa seca (BS) acima do solo de espécies florestais cultivadas em quatro sistemas agroflorestais (SAF) em Cametá, Pará.

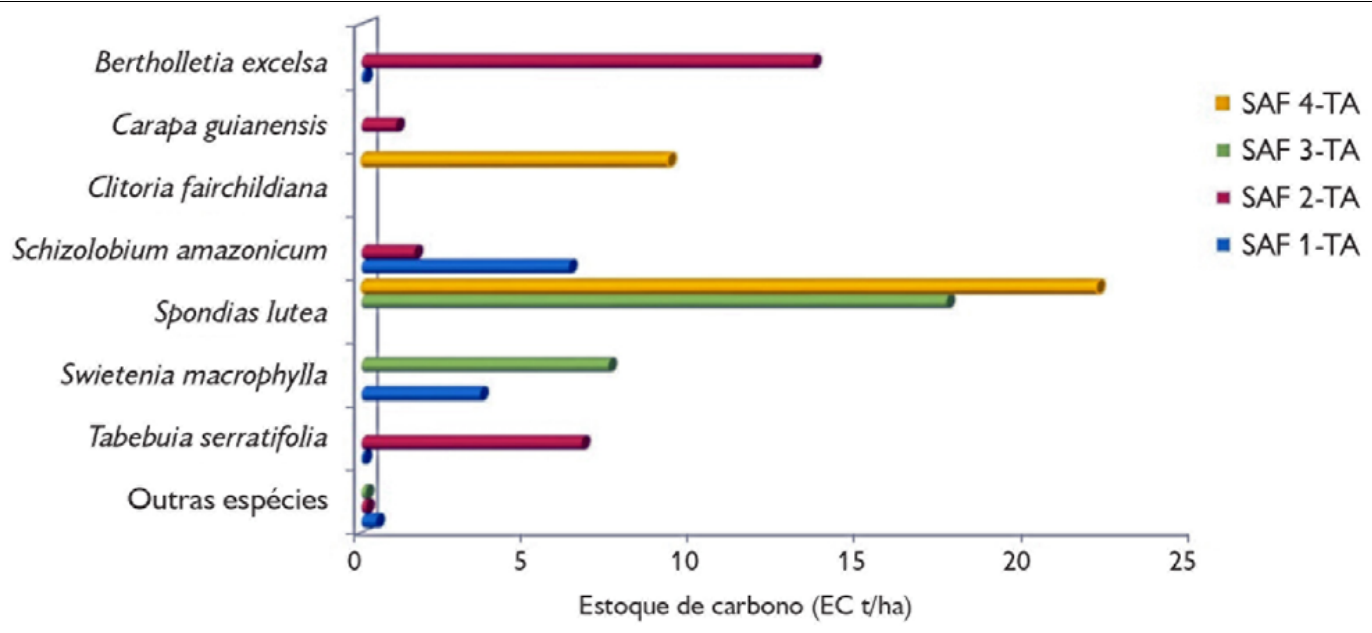

Figura 3. Estoque de carbono (EC) na biomassa seca (BS) acima do solo de espécies florestais cultivadas em quatro sistemas agroflorestais (SAF) em Tomé-Açu, Pará.

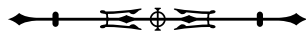


A média de EC nos SAF-CM foi mais que o dobro da média encontrada nos SAF-TA. Essa diferença pode estar associada ao regime de manejo adotado, que, no caso dos SAF-CM, é moderado, preservando maior número de árvores, componente que aporta mais biomassa e, logo, estoca mais carbono. Poderíamos dizer também que, além da abundância das árvores, há outras variáveis que podem influenciar o desenvolvimento vegetativo e a produção de biomassa de um ecossistema, como o tamanho (DAP e H) das plantas e o tempo de plantio e/ou de manejo.

Os SAF-CM e SAF-TA estudados estocaram por ano, em média, 4,94 Mg C ha- ano-1 e 2,49 Mg C ha-1 ano-1, respectivamente, ou seja, a cada ano, no mínimo, 2 a $5 \mathrm{Mg}$ de $\mathrm{CO}_{2}$ são extraídas da atmosfera (Tabela 4), sem contar o EC na biomassa microbiana, nas raízes e no solo. Em ambos os casos, as maiores médias anuais de EC foram observadas nos SAF mais jovens, onde as atividades fisiológicas são mais intensas, com maior incremento em biomassa.

A média de EC encontrada nos SAF-CM foi superior às médias observadas em quatro ecossistemas antrópicos, mais do que o dobro das pastagens (PAS) de Anajatuba, no Maranhão, e 80,3\% a mais do que os consórcios de árvores (CAR) de Porto Velho, em Rondônia. Ela, no entanto, foi inferior ao quintal agroflorestal (QAF) de Arari, no Maranhão, apresentando 69,2\% do estocado nesse ambiente e 76,6\% do monocultivo de árvores (MCA) de Manaus, Amazonas (Tabela 2).

Em relação aos ecossistemas antropizados, a média de EC nos SAF-CM foi superior em três médias de EC, destaque aos $87,3 \%$ a mais do que a média de EC das capoeiras (CAP) de Parauapebas, no Pará. Essa média, contudo, foi inferior a três médias de EC, com $64,2 \%$ do EC das capoeiras enriquecidas (CAE) de Paragominas, no Pará. Em relação aos ecossistemas naturais, esse dado foi inferior a todas as médias dos sistemas, estocando 29,8\% do EC das florestas de terra firme (FTF) da Amazônia e pouco mais da metade $(51,9 \%)$ da floresta de várzea (FVA) de Cametá, no Pará (Tabela 2).

A média de EC encontrada nos SAF-TA, por sua vez, foi inferior às médias de todos os estudos nas três categorias, tendo, entretanto, estocado $92,5 \%$ do EC das pastagens (PAS) de Anajatuba, no Maranhão, em ecossistemas antrópicos, 50,5\% das capoeiras enriquecidas (CAE) de Igarapé-Açu, no Pará, em ecossistemas antropizados, e 12,5\% das florestas de terra firme (FTF) da Amazônia nos ecossistemas naturais (Tabela 2).

Os SAF podem ser considerados como uma alternativa viável do ponto de vista da resiliência ambiental e da segurança alimentar, devido à diversidade registrada entre os sistemas, as estruturas e as espécies componentes, bem como às práticas de manejo adotadas pelos produtores. Isso também é justificável se levarmos em consideração que os SAF, no amadurecimento, atingem EC similar ou até superior ao de outros sistemas de uso da terra, onde poucas culturas agrícolas ou nenhuma apresentam EC e produzem alimentos ao mesmo tempo e no mesmo espaço, como ocorre com os SAF estudados.

\section{CONCLUSÕES}

Nos SAF estudados em Cametá (SAF-CM) e Tomé-Açu (SAF-TA), E. oleracea e T. cacao mereceram destaque, devido à sua importância socioeconômica, em razão de produção voltada para consumo e venda, e ambiental, tendo em vista a produção de biomassa e de carbono, o que as credencia para compor SAF e outros sistemas produtivos nesses municípios e na Amazônia.

O EC foi maior nas árvores tanto nos SAF-CM - com destaque para $C$. guianensis, V. surinamensis e H. brasiliensis - quanto nos SAF-TA - com destaque para S. lutea e B. excelsa. Essas espécies podem ser recomendadas para compor SAF na região estudada. O manejo adotado nos SAF influenciou a riqueza de espécies e o EC. Desse modo, os SAF-CM mostraram mais tendências naturais, enquanto os SAF-TA evidenciaram mais antropia.

Os percentuais de espécies que servem para consumo e para venda, bem como a média de EC por ano, nos SAFCM e SAF-TA, monstraram, ao mesmo tempo, viabilidade socioeconômica e ambiental em ambas as áreas de estudo. 


\section{AGRADECIMENTOS}

Agradeço a Deus acima de tudo. À confiança e à colaboração dos proprietários das áreas onde foram realizados os estudos: Sr. Bráulio Leão (Zito), Sr. Raimundo Lousada, Sr. Wilson Pinto e Sra. Maria Amélia, e suas famílias, em Cametá, e Sr. Michinori Konagano e Sr. José Maria Mendes, e suas famílias, em Tomé-Açu.

\section{REFERÊNCIAS}

BARTELT, D., J. KOCH\& M. M. TOURINHO, 2000. Anbau von Acai (Euterpe oleracea) und Kakao (Theobroma sylvestre) in Primärwäldern der varzeas am rio Tocantins (Brasilien/Para). Forstarchiv 71(6): 250-256.

BENTES-GAMA, M. M., J. R. V. GAMA \& M. M. TOURINHO, 1999. Huertos caseros em la comunidad ribereña de Villa Cuera, em el município de Bragança em el Nordeste Paraense. Agroforesteria em las Américas 6(24): 8-12.

BOLFE, E. L., M. C. FERREIRA \& M. BATISTELLA, 2009. Biomassa epígea e estoque de carbono de agroflorestas em Tomé-Açu, PA. Revista Brasileira de Agroecologia 4(2): 2171-2175.

BOLFE, E. L., M. BATISTELLA \& M. C. FERREIRA, 2012. Correlação de variáveis espectrais e estoque de carbono da biomassa aérea de sistemas agroflorestais. Pesquisa Agropecuária Brasileira 47(9): 1261-1269. DOI: http://dx.doi.org/10.1590/S0100204X2012000900011.

BRANCHER, T., 2010. Estoque e ciclagem de carbono em sistemas agroflorestais em Tomé-Açu, Amazônia oriental: 1-58. Dissertação (Mestrado em Ciências Ambientais) - Universidade Federal do Pará, Belém.

BRIENZA JÚNIOR, S., M. DENICH, H. FOELSTER \& P. L. G. VLEK, 2001. Enriquecimiento de barbechos con leguminosas arbóreas como alternativa para la tumba y quema en la Amazonía Oriental Brasileña. Agroforestería en las Américas 8(32): 16-19.

BRIENZA JÚNIOR, S., 2012. Enriquecimento de florestas secundárias como tecnologia de produção sustentável para a agricultura familiar. Boletim do Museu Paraense Emílio Goeldi. Ciências Naturais 7(3): 331-337.

BROWER, J. E., J. H. ZAR \& C. N. ENDE, 1998. Field and laboratory methods for general ecology: 1-273. McGraw, New York.

ChaVES, M. S., A. C. CAETANO, R. L. G. MACEDO \& R. C. M. MEIRELES, 2011. Imobilização de carbono em um castanhal no IFAM. Cadernos de Agroecologia 6(2): 1-5. Disponível em: <http://revistas.aba-agroecologia.org.br/index.php/cad/article/ view/10690>. Acesso em: 2 maio 2018.
COSTA, L. G. S., I. S. MIRANDA, M. GRIMALDI, M. L. SILVA, D. MITJA \& T. T. S. LIMA, 2012. Biomass in different types of land use in the Brazil's 'arc of deforestation'. Forest Ecology and Management 278: 101-109. DOI: http://dx.doi.org/10.1016/j.foreco.2012.04.007.

DALLAGNOL, F. S., F. MOGNON, C. R. SANQUETTA \& A. P. D. CORTE, 2011. Teores de carbono de cinco espécies florestais e seus compartimentos. Floresta e Ambiente 18(4): 410-416. DOI: http:// dx.doi.org/10.4322/floram.2011.060.

DIAS, I. P., B. C. COSTA, M. M. TOURINHO \& J. F. ALMEIDA, 2015. Avaliação da estimativa de fixação de carbono em sistemas agroflorestais na região amazônica. Revista Verde 10(5): 7-10. DOI: https://doi.org/10.18378/rvads.v10i5.3411.

DUBOIS, J. C. L., 1996. Manual agroflorestal para a Amazônia: v. 1: 1-228. REBRAF, Rio de Janeiro.

FASSBENDER, H. W., 1993. Modelos edafológicos de los sistemas de produción agroforestales: 2. ed.: 1-29. Centro Agronomico Tropical de Investigacion y Enseñanza, Turrialba.

FEARNSIDE, P. M., 1996. Amazonian deforestation and global warming: carbon stocks in vegetation replacing Brazil's Amazon forest. Forest Ecology and Management 80(1-3): 21-34. DOI: https://doi.org/10.1016/0378-1127(95)03647-4.

HAIRIAH, K., S. M. SITOMPULL, M. NOORDWIJK \& C. PALM, 2001. Methods for sampling carbon stocks above and below ground. In: M. NOORDWIJK, S. WILLIAMS \& B. VERBIST (Ed.): Towards integrated natural resource management in forest margins of the humid tropics: local action and global concerns: 1-49. ICRAF, Bogoi.

HIGUCHI, N. \& A. J. CARVALHO JR., 1994. Fitomassa e conteúdo de carbono de espécies arbóreas da Amazônia. Anais do Seminário Emissão e Sequestro de $\mathrm{CO}_{2}$ - uma nova oportunidade de negócios para o Brasil 125-153.

HIGUCHI, N., J. SANTOS, R. J. RIBEIRO, L. MINETTE \& Y. BIOT, 1998. Biomassa da parte aérea da vegetação de floresta tropical úmida de terra-firme da Amazônia brasileira. Acta Amazonica 28(2): 153-165. DOI: http://dx.doi.org/10.1590/1809-43921998282166.

HOMMA, A. K. O., R. T. WALKER, R. A. CARVALHO, C. A. P. FERREIRA, A. J. CONTO, A. I. M. SANTOS \& F. N. SCATENA, 1995. Dinâmica dos sistemas agroflorestais: o caso dos agricultores nipo-brasileiros em Tomé-Açu-PA. In: J. M. M. COSTA (Org.): Amazônia. Desenvolvimento econômico, desenvolvimento sustentável e sustentabilidade de recursos naturais - Pará: 37-56. UFPA-NUMA, Belém.

HOMMA, A. K. O., 2006. Organização da produção e comercialização de produtos agropecuários: o caso da colônia agrícola nipo-brasileira de Tomé-Açu, Pará. In: L. J. M. VILCAHUAMÁN, J. RIBASKI \&A. M. B. MACHADO (Ed.): Sistemas agroflorestais e desenvolvimento com proteção ambiental: perspectivas, análise e tendências: 51-77. EMBRAPA Florestas, Colombo.

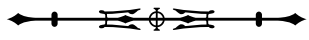


INSTITUTO BRASILEIRO DE GEOGRAFIA E ESTATÍSTICA(IBGE), 2012. Censo demográfico 2010: 1-211. IBGE, Rio de Janeiro.

KATO, O. R., M. S. A. KATO, C. J. R. CARVALHO, R. O. FIGUEIREDO, A. CAMARÃO, T. D. A. SÁ, M. DENICH \& K. VIELHAUER, 2006. Uso de agroflorestas no manejo de florestas secundárias. Anais do Congresso Brasileiro de Sistemas Agroflorestais - CBSAF 6: 119-138.

KATO, O. R., M. K. SHIMIZU, A. C. M. R. BORGES, C. M. B. C. AZEVEDO, J. S. L. OLIVEIRA, S. S. VASCONCELOS \& T. D. A. SÁ, 2012. Desenvolvimento da produção de frutas em sistemas agroflorestais no estado do Pará. Congresso Brasileiro de Fruticultura 22: 1-14.

LOCATELLI, M., E. P. SILVA, A. H. VIEIRA, V. F. SOUZA \& R. S. MACEDO, 2004. Cultivo de cupuaçu (Theobroma grandiflorum Schum) consorciado com espécies florestais em Machadinho d'Oeste, Rondônia - característica de solo. Agrossilvicultura 1(1): 101-105.

MATOS, F. D. A. \& F. F. KIRCHNER, 2008. Estimativa de biomassa da floresta ombrófila densa de terra firme na Amazônia Central com o satélite Ikonos II. Floresta 38(1): 157-171.

MAVISOY, H. M., 2013. Estoques de carbono em floretas, capoeiras e sistemas agroflorestais da Amazônia oriental: 1-64. Dissertação (Mestrado em Agroecologia) - Universidade Estadual do Maranhão, São Luís.

MONTAGNINI, F. \& P. K. R. NAIR, 2004. Carbon sequestration: an underexploited environmental benefit of agroforestry systems. Agroforestry Systems 61: 281-295. DOI: https://doi.org/10.1023/ B:AGFO.0000029005.92691.79.

MURILLO, M. A., 1997. Almacienamiento y fijación de Carbono en ecosistemas forestales. Revista Forestal Centroamericana 6(19): 9-12.

OLIVEIRA, J. S. R. \& O. R. KATO, 2010. Território de exceção: experiências do agricultor inovador Pedro Araújo e família na comunidade de Monte Sião: 1-19. APEPA, São Domingos do Capim.

OSTERROOHT, von M., 2002. Manejo de Sistemas Agroflorestais - SAF's. Agroecologia 15: 12-13.

PEREIRA, C. P., 2001. Avaliação da biomassa acumulada em áreas de vegetação secundária "capoeira" enriquecida com árvores leguminosas, no nordeste do estado do Pará: 1-36. Dissertação (Mestrado em Ciências Florestais) - Faculdade de Ciências Agrárias do Pará, Belém.
PORRO, R., R. P. MILLER, M. R. TITO, J. A. DONOVAN, J. L. VIVAN, R. TRANCOSO, R. F. VAN KANTEN, J. E. GRIJALVA, B. L. RAMIREZ \& A. L. GONÇALVES, 2012. Agroforestry in the Amazon region: a pathway for balancing conservation and development. In: P. K. R. NAIR \& D. GARRITY (Ed.): Agroforestry: the future of global land use: 391-428. Springer (Advances in Agroforestry, 9), Dordrecht.

QUEIROZ, J. A. L. \& S. MOCHIUTTI, 2000. Diversidade florestal em sistemas agroflorestais, com açaizeiro no estuário amazônico: 147-149. EMBRAPA Amazônia Ocidental, Manaus.

SANTOS, S. R. M., I. S. MIRANDA \& M. M. TOURINHO, 2004. Estimativa de biomassa de sistemas agroflorestais das várzeas do rio Juba, Cametá, Pará. Acta Amazonica 34(1): 1-8. DOI: http://dx.doi. org/10.1590/S0044-59672004000100001.

SMITH, N., J. DUBOIS, D. CURRENT, E. LUTZ \& C. CLEMENT, 1998. Agroforestry experiences in the Brazilian Amazon: constraints and opportunities: 1-146. Pilot Programme to Conserve the Brazilian Rain Forest/World Bank, Brasília.

SOUGATA, B. \&J. SHIBU, 2012. Agroforestry for biomass production and carbon sequestration: an overview. Agroforesty Systems 86(2): 105-111. DOI: https://doi.org/10.1007/s10457-012-9573-X.

THIBAU, C. E., 2000. Produção sustentada em florestas: conceitos e tecnologias, biomassa energética, pesquisas e constatações: 1-512. CVRD, Belo Horizonte.

TSUCHIYA, A. \& M. HIRAOKA, 1999. Forest biomass and wood consumption in the lower course of the Amazon: a case study of the Urubuera Island. Acta Amazonica 29(1): 79-95.

VIEIRA, T. A., L. S. ROSA, P. C. S. VASCONCELOS, M. M. SANTOS \& R. S. MODESTO, 2007. Sistemas agroflorestais em áreas de agricultores familiares em Igarapé-Açu, Pará: caracterização florística, implantação e manejo. Acta Amazonica 37(4): 549-557. DOI: http://dx.doi.org/10.1590/S0044-59672007000400010.

VILLA, P. M., S. V. MARTINS, L. MONSANTO, S. N. OLIVEIRANETO \& N. M. CANCIO, 2015. La agroforestería como estrategia para la recuperación y conservación de reservas de carbono en bosques de la Amazonía. Bosque 36(3): 347-356. DOI: http:// dx.doi.org/10.4067/S0717-92002015000300002. 